PROCEEDINGS OF THE

AMERICAN MATHEMATICAL SOCIETY

Volume 131, Number 11, Pages 3387-3393

S 0002-9939(03)06912-0

Article electronically published on February 24, 2003

\title{
THE HOCHSCHILD COHOMOLOGY RING OF A SELFINJECTIVE ALGEBRA OF FINITE REPRESENTATION TYPE
}

\author{
EDWARD L. GREEN, NICOLE SNASHALL, AND ØYVIND SOLBERG \\ (Communicated by Martin Lorenz)
}

\begin{abstract}
This paper describes the Hochschild cohomology ring of a selfinjective algebra $\Lambda$ of finite representation type over an algebraically closed field $K$, showing that the quotient $\mathrm{HH}^{*}(\Lambda) / \mathcal{N}$ of the Hochschild cohomology ring by the ideal $\mathcal{N}$ generated by all homogeneous nilpotent elements is isomorphic to either $K$ or $K[x]$, and is thus finitely generated as an algebra. We also consider more generally the property of a finite dimensional algebra being selfinjective, and as a consequence show that if all simple $\Lambda$-modules are $\Omega$-periodic, then $\Lambda$ is selfinjective.
\end{abstract}

\section{INTRODUCTION}

This paper describes the Hochschild cohomology ring of a selfinjective algebra of finite representation type over an algebraically closed field.

Throughout this paper let $K$ be an algebraically closed field, and let $\Lambda$ be a finite dimensional $K$-algebra. All modules are finitely generated right modules unless otherwise stated, and $\bmod \Lambda$ denotes the category of all finitely generated right $\Lambda$ modules. Denote by $\Lambda^{e}$ the enveloping algebra $\Lambda^{\mathrm{op}} \otimes_{K} \Lambda$. Then $\Lambda$ may be considered as a right $\Lambda^{e}$-module, or equivalently as a $\Lambda, \Lambda$-bimodule. The Hochschild cohomology ring $\operatorname{HH}^{*}(\Lambda)$ may be described by $\operatorname{HH}^{*}(\Lambda)=\operatorname{Ext}_{\Lambda^{e}}^{*}(\Lambda, \Lambda)=\bigoplus_{i \geq 0} \operatorname{Ext}_{\Lambda^{e}}^{i}(\Lambda, \Lambda)$ with the Yoneda product.

The selfinjective algebras of finite representation type over an algebraically closed field were classified by C. Riedtmann [11]. She showed that one can associate to any such algebra one of the Dynkin diagrams $A_{n}, D_{n}, E_{6}, E_{7}$ or $E_{8}$, that is, the tree class of the stable Auslander-Reiten quiver of the algebra. H. Asashiba has subsequently shown in [1] that derived equivalence and stable equivalence coincide for selfinjective algebras of finite representation type over an algebraically closed field (see also [10] for the special case of type $A_{n}$ ).

For type $A_{n}$ these algebras fall into two classes, denoted wreath-like algebras and Möbius algebras. The selfinjective Nakayama algebras represent the distinct derived equivalence classes of the wreath-like algebras. K. Erdmann and T. Holm showed in [5], for such an algebra $\Lambda$, that the syzygy $\Omega_{\Lambda^{e}}^{2}(\Lambda)$ of a minimal projective bimodule resolution of $\Lambda$ is isomorphic to a twisted bimodule structure on $\Lambda$, that is, to ${ }_{1} \Lambda_{\sigma}$ where $\sigma$ is an automorphism of $\Lambda$ of finite order. Recall, for an algebra

Received by the editors December 5, 2001 and, in revised form, June 17, 2002.

2000 Mathematics Subject Classification. Primary 16E40, 16G10, 16G60.

(C)2003 American Mathematical Society 
automorphism $\sigma$ of $\Lambda$, that the underlying vector space of ${ }_{1} \Lambda_{\sigma}$ is $\Lambda$, the action from the left is multiplication in $\Lambda$, but the right multiplication is defined as $\lambda * x=$ $\lambda \sigma^{-1}(x)$. In [6] it was shown for the Möbius algebras $B=B_{m, k}$ (as given in [12], and which represent the distinct stable equivalence classes and also the distinct derived equivalence classes of Möbius algebras) that $\Omega_{B^{e}}^{2 k-1}(B) \simeq{ }_{1} B_{\sigma}$ where $\sigma$ is an automorphism of $B$ of finite order. Thus the representatives of the distinct derived equivalence classes of selfinjective algebras of finite representation type $A_{n}$ each have a periodic projective bimodule resolution.

In this paper we generalise these results to show, for any selfinjective algebra $\Lambda$ of finite representation type, that there is some natural number $n$ and some automorphism $\sigma$ of $\Lambda$ such that $\Omega_{\Lambda^{e}}^{n}(\Lambda) \simeq{ }_{1} \Lambda_{\sigma}$.

Since Hochschild cohomology is invariant under derived equivalence, the study in [5] and [6] of the Hochschild cohomology ring provides information for all selfinjective algebras of finite representation type $A_{n}$ over an algebraically closed field.

In Theorem 1.6 we study, for certain algebras $\Lambda$, the structure of $\mathrm{HH}^{*}(\Lambda) / \mathcal{N}$ where $\mathcal{N}$ is the ideal in $\operatorname{HH}^{*}(\Lambda)$ generated by the homogeneous nilpotent elements. As a corollary we show, for a finite dimensional selfinjective indecomposable $K$ algebra $\Lambda$ of finite representation type, that $\mathrm{HH}^{*}(\Lambda) / \mathcal{N}$ is isomorphic to either $K$ or $K[x]$.

\section{Main Results}

Let $\Lambda$ be a finite dimensional $K$-algebra. For several classes of selfinjective algebras it is known that $\Lambda$ is an $\Omega$-periodic $\Lambda^{e}$-module, that is, $\Omega_{\Lambda^{e}}^{n}(\Lambda) \simeq \Lambda$ for some $n$, and hence it follows that every indecomposable $\Lambda$-module is $\Omega$-periodic or projective. For a group algebra over a finite group with char $K=p$ prime, it is well known that every indecomposable module is $\Omega$-periodic or projective if and only if the Sylow $p$-subgroups are cyclic or $p=2$ and the Sylow 2-subgroups are generalised quaternion. For finite dimensional selfinjective algebras, even for those of finite representation type, it is unknown if $\Lambda$ is an $\Omega$-periodic $\Lambda^{e}$-module. However, it was observed by M. C. R. Butler that if a finite dimensional algebra $\Lambda$ is $\Omega$-periodic as a $\Lambda^{e}$-module, then $\Lambda$ is selfinjective. A proof of this statement is also included within Theorem 1.4 .

Now let $\Lambda$ be a finite dimensional selfinjective $K$-algebra. Then it is well known that $\Lambda^{e}$ is also a finite dimensional selfinjective algebra. Moreover $\Lambda$ as a $\Lambda^{e}$-module is indecomposable if and only if $\Lambda$ is an indecomposable algebra. These observations lead to the following result.

Proposition 1.1. Let $\Lambda$ be a finite dimensional indecomposable algebra over an algebraically closed field $K$. Assume that $\Omega_{\Lambda^{e}}^{i}(\Lambda) \simeq \Lambda$ as $\Lambda^{e}$-modules for some $i$. Then $\mathrm{HH}^{*}(\Lambda) / \mathcal{N} \simeq K[x]$, where $x$ is of degree $n$ with $n$ being the minimal $i$ such that $\Omega_{\Lambda^{e}}^{i}(\Lambda) \simeq \Lambda$ as $\Lambda^{e}$-modules and $\mathcal{N}$ is the ideal in $\mathrm{HH}^{*}(\Lambda)$ generated by the homogeneous nilpotent elements.

Using the above observations the proof of this result is a direct consequence of Proposition 1.3 below, which is a result due to J. Carlson (see Proposition 5.10.2 in [3]). For the convenience of the reader we include a proof of Proposition 1.3. Before doing that we point out the following consequences.

For a selfinjective Nakayama algebra $\Lambda$ we have from [5] that $\Omega_{\Lambda^{e}}^{2}(\Lambda)$ is isomorphic to ${ }_{1} \Lambda_{\tau}$ for some automorphism $\tau$ of finite order. For the Möbius algebras $B_{m, k}$, 
it was shown in [6] that $\Omega_{\Lambda^{e}}^{2 k-1}(\Lambda)$ is isomorphic to ${ }_{1} \Lambda_{\tau}$ for some automorphism $\tau$ of finite order. Details of the automorphism $\tau$ for each algebra are given in [5] and [6] so that $n$ may be chosen minimal with $\Omega_{\Lambda^{e}}^{n}(\Lambda) \simeq \Lambda$ as $\Lambda^{e}$-modules. From [7], [8] $\Omega_{\Lambda^{e}}^{6}(\Lambda) \simeq \Lambda$ for every finite dimensional preprojective algebra $\Lambda$. It is of interest to note that almost all of the finite dimensional preprojective algebras are of wild representation type. Thus by Proposition 1.1 we immediately obtain the following.

Corollary 1.2. Let $\Lambda$ be a finite dimensional algebra over an algebraically closed field $K$. Then $\mathrm{HH}^{*}(\Lambda) / \mathcal{N} \simeq K[x]$ where $\mathcal{N}$ is the ideal in $\mathrm{HH}^{*}(\Lambda)$ generated by the homogeneous nilpotent elements if

(i) $\Lambda$ is a selfinjective Nakayama algebra.

(ii) $\Lambda$ is a Möbius algebra.

(iii) $\Lambda$ is a preprojective algebra.

Proposition 1.3. Let $K$ be an algebraically closed field, and let $\Lambda$ be a finite dimensional selfinjective $K$-algebra. Let $M$ be an indecomposable $\Lambda$-module. Suppose $M \simeq \Omega_{\Lambda}^{i}(M)$ for some $i \geq 1$, and let $n \geq 1$ be the minimal such $i$. Then $\operatorname{Ext}_{\Lambda}^{*}(M, M) / \mathcal{N} \simeq K[x]$, where $\mathcal{N}$ is the ideal generated by the homogeneous nilpotent elements in $\operatorname{Ext}_{\Lambda}^{*}(M, M)$ and $x$ is a homogeneous element in degree $n$.

Proof. Let $z: \Omega_{\Lambda}^{s}(M) \rightarrow M$ in $\operatorname{Ext}_{\Lambda}^{s}(M, M)$ be a nilpotent element. Let $\gamma: \Omega_{\Lambda}^{m}(M)$ $\rightarrow M$ be any element in $\operatorname{Ext}_{\Lambda}^{m}(M, M)$. First we prove that $\gamma z$ is again a nilpotent element in $\operatorname{Ext}_{\Lambda}^{*}(M, M)$. We have that $z \Omega_{\Lambda}^{s}(\gamma): \Omega_{\Lambda}^{m+s}(M) \rightarrow M$. Choose $r$ such that $r(m+s)=q n$ for some $q$. Then $\alpha=\left(z \Omega_{\Lambda}^{s}(\gamma)\right)^{r}: \Omega_{\Lambda}^{q n}(M) \simeq M \rightarrow M$. If $\alpha$ is an isomorphism, then $z$ is a split epimorphism. Since $M$ and $\Omega_{\Lambda}^{s}(M)$ are indecomposable, $z$ is an isomorphism. This is impossible, since $z$ is a nilpotent element. Since $\operatorname{End}_{\Lambda}(M)$ is a local ring, it follows that $\alpha$ is a nilpotent element. Now considering the liftings $\Omega_{\Lambda}^{i n}(\alpha)$ of $\alpha$ and using that $\alpha$ is nilpotent, the liftings $\Omega_{\Lambda}^{i n}(\alpha)$ cannot be an isomorphism for any $i \geq 0$. Hence $\Omega_{\Lambda}^{i n}(\alpha): M \rightarrow M$ are nilpotent elements in $\operatorname{End}_{\Lambda}(M)$ for all $i \geq 0$ and therefore also elements in the radical of $\operatorname{End}_{\Lambda}(M)$. Let $l$ be the radical length of $\operatorname{End}_{\Lambda}(M)$. Then it follows that $\alpha^{l}=0$ in $\operatorname{Ext}_{\Lambda}^{*}(M, M)$. Hence $\gamma z$ is a nilpotent element in $\operatorname{Ext}_{\Lambda}^{*}(M, M)$. Using injective resolutions a similar proof shows that $z \gamma$ is nilpotent.

Next we let $s \neq q n$ for all $q$, and show that any element $z: \Omega_{\Lambda}^{s}(M) \rightarrow M$ is a nilpotent element in $\operatorname{Ext}_{\Lambda}^{*}(M, M)$. Choose $r$ such that $r s=q n$ for some $q$. Let $\alpha=z^{r}$. As above, $\alpha$ cannot be an isomorphism, since otherwise $z$ would have to be an isomorphism. Hence $\alpha$ as an element in $\operatorname{End}_{\Lambda}(M)$ is nilpotent. It follows as above that $\alpha$ is nilpotent as an element in $\operatorname{Ext}_{\Lambda}^{*}(M, M)$. Hence $z$ is nilpotent in $\operatorname{Ext}_{\Lambda}^{*}(M, M)$.

Finally let $x: \Omega_{\Lambda}^{n}(M) \simeq M \rightarrow M$ be represented by the identity map from $M$ to $M$. Then $x$ is clearly not a nilpotent element in $\operatorname{Ext}_{\Lambda}^{*}(M, M)$. Suppose $x^{t}$ for some $t$ is in the ideal $\mathcal{N}$ generated by the homogeneous nilpotent elements in $\operatorname{Ext}_{\Lambda}^{*}(M, M)$. Assume that $x^{t}=\sum_{i} r_{i} n_{i} s_{i}$ with $r_{i}$ and $s_{i}$ in $\operatorname{Ext}_{\Lambda}^{*}(M, M)$ and $n_{i}$ a homogeneous nilpotent element in $\operatorname{Ext}_{\Lambda}^{*}(M, M)$ for all $i$. We can also assume that $r_{i}$ and $s_{i}$ are homogeneous elements. By the above, all the elements $r_{i} n_{i} s_{i}$, when viewed in $\operatorname{End}_{\Lambda}(M)$, are nilpotent elements. Therefore the sum $\sum_{i} r_{i} n_{i} s_{i}$ is a nilpotent element in $\operatorname{Ext}_{\Lambda}^{*}(M, M)$. This is a contradiction, so that any power of $x$ is not in the ideal $\mathcal{N}$. It follows from this that $\operatorname{Ext}_{\Lambda}^{*}(M, M) / \mathcal{N} \simeq K[x]$, since $\operatorname{End}_{\Lambda}(M) / \operatorname{rad}_{\operatorname{End}}(M) \simeq K$. 
Our main concern in this paper is the study of finite dimensional selfinjective $K$-algebras of finite representation type. For such an algebra $\Lambda$ there exists a natural number $n$ and an automorphism $\sigma$ of $\Lambda$ such that $\Omega_{\Lambda^{e}}^{n}(\Lambda) \simeq{ }_{1} \Lambda_{\sigma}$. This fact was noted by S. Brenner and M. C. R. Butler in [4]. The next result looks more generally at this condition. The authors are grateful to K. Erdmann for her helpful comments on previous versions of this paper which led to the following more general formulation of Theorem 1.4 .

Theorem 1.4. Let $K$ be an algebraically closed field, and let $\Lambda$ be a finite dimensional indecomposable $K$-algebra. Then the following statements are equivalent:

(a) All simple $\Lambda$-modules are $\Omega$-periodic.

(b) There exists a natural number $n$ and an automorphism $\sigma$ of $\Lambda$ such that $\Omega_{\Lambda^{e}}^{n}(\Lambda) \simeq{ }_{1} \Lambda_{\sigma}$, and $\sigma\left(e_{i}\right)=e_{i}$ for a complete set of orthogonal idempotents $\left\{e_{i}\right\}$.

Moreover, if $\Lambda$ satisfies these conditions, then $\Lambda$ is selfinjective.

Proof. First we note that the minimal projective resolution $P^{*}$ of $\Lambda$ over $\Lambda^{e}$ gives rise to a minimal projective resolution of any simple $\Lambda$-module $S$ by applying the functor $S \otimes_{\Lambda}-$ (see $\left.[9]\right)$.

Now suppose that all simple right $\Lambda$-modules are $\Omega$-periodic. Let $n \geq 1$ be minimal such that $\Omega_{\Lambda}^{n}\left(S_{i}\right) \simeq S_{i}$ for all simple $\Lambda$-modules $\left\{S_{i}\right\}$.

Let $B=\Omega_{\Lambda^{e}}^{n}(\Lambda)$. Since $\Omega_{\Lambda^{e}}^{i}(X)$ is a projective left $\Lambda$-module for any $i \geq 0$ and any $\Lambda^{e}$-module $X$ which is also a projective left $\Lambda$-module, we may take $X=\Lambda$, and so $B$ is a projective left $\Lambda$-module. Thus $-\otimes_{\Lambda} B: \bmod \Lambda \rightarrow \bmod \Lambda$ is an exact functor. Since $S \otimes_{\Lambda} B \simeq S$ for all simple right $\Lambda$-modules $S$, it follows by induction on the length of a module that $l\left(M \otimes_{\Lambda} B\right)=l(M)$ for all finitely generated $\Lambda$-modules $M$.

We next show that $P \otimes_{\Lambda} B \simeq P$ for all projective right $\Lambda$-modules $P$. Let $P$ be an indecomposable projective module. The exact sequence $0 \rightarrow P \mathfrak{r} \rightarrow P \rightarrow P / P \mathfrak{r} \rightarrow 0$ induces the exact sequence $0 \rightarrow P \mathfrak{r} \otimes_{\Lambda} B \rightarrow P \otimes_{\Lambda} B \rightarrow P / P \mathfrak{r} \rightarrow 0$. The module $P \otimes_{\Lambda} B$ is a projective $\Lambda$-module with $l\left(P \otimes_{\Lambda} B\right)=l(P)$. Moreover, $P / P \mathfrak{r}$ is in the top of $P \otimes_{\Lambda} B$; hence $P$ is a direct summand of $P \otimes_{\Lambda} B$. We infer from this that $P \otimes_{\Lambda} B \simeq P$ by a length argument. This implies that there is an isomorphism $\theta: \Lambda \otimes_{\Lambda} B \rightarrow \Lambda$. Defining $\chi: B \rightarrow \Lambda$ by letting $\chi(b)=\theta(1 \otimes b)$ shows that $B$ as a right $\Lambda$-module is isomorphic to $\Lambda$.

Now consider a simple left $\Lambda$-module $T$. Since $B$ is isomorphic to $\Lambda$ as a right $\Lambda$-module, $B \otimes_{\Lambda} T \simeq T$ as a vector space over $K$. For any simple right $\Lambda$-module $S$ we know, from above, that $S \otimes_{\Lambda} B \otimes_{\Lambda} T \simeq S \otimes_{\Lambda} T$ and this is non-zero if and only if $S \simeq D(T)$. Then $\Lambda / \mathfrak{r} \otimes_{\Lambda} B \otimes_{\Lambda} T \simeq T$ and hence the top of $B \otimes_{\Lambda} T$ is isomorphic to $T$ as left $\Lambda$-modules. Now by a dimension argument we have that $B \otimes_{\Lambda} T \simeq T$ as left $\Lambda$-modules for all simple left $\Lambda$-modules $T$. As above, it then follows that $B$ is isomorphic to $\Lambda$ as a left $\Lambda$-module.

Up to isomorphism, $\left\{\Lambda e_{i} \otimes_{K} e_{j} \Lambda\right\}_{i, j}$ are all the isomorphism classes of indecomposable projective $\Lambda^{e}$-modules, where $\left\{e_{i}\right\}_{i=1}^{N}$ is a complete set of primitive orthogonal idempotents for $\Lambda$. Given the choice of $n$ it follows that $P^{n} \simeq \bigoplus_{i=1}^{N} \Lambda e_{i} \otimes_{K} e_{i} \Lambda$. Let $\varphi: P^{n} \rightarrow B$ be the map in the minimal projective resolution of $\Lambda$ over $\Lambda^{e}$, and let $b=\varphi\left(\sum_{i=1}^{N} e_{i} \otimes e_{i}\right)$. Then $e_{i} b=b e_{i}$ for all $i$. Using that $B$ is isomorphic to $\Lambda$ as a left and right $\Lambda$-module, we tensor $\varphi$ with $\Lambda / \mathfrak{r}$ to give surjective maps $\bigoplus_{i=1}^{N} \Lambda e_{i} \otimes_{K} e_{i} \Lambda / e_{i} \mathfrak{r} \rightarrow \Lambda / \mathfrak{r}$ and $\bigoplus_{i=1}^{N} \Lambda e_{i} / \mathfrak{r} e_{i} \otimes_{K} e_{i} \Lambda \rightarrow \Lambda / \mathfrak{r}$. It follows that 
$\varphi\left(e_{i} \otimes e_{i}\right) \notin \mathfrak{r} B$ and $\varphi\left(e_{i} \otimes e_{i}\right) \notin B \mathfrak{r}$. Hence $\Lambda \varphi\left(e_{i} \otimes e_{i}\right) \simeq \Lambda e_{i}$ and $\varphi\left(e_{i} \otimes e_{i}\right) \Lambda \simeq e_{i} \Lambda$ for all $i$. Thus $\Lambda b=B=b \Lambda$.

By length arguments the map $\psi: \Lambda \rightarrow B$ given by $\lambda \mapsto \lambda b$ is an isomorphism of left $\Lambda$-modules. Then $\psi(1)=b$. Define $\sigma: \Lambda \rightarrow \Lambda$ by letting $\sigma(\gamma)=\psi^{-1}(b \gamma)$. Then $b \gamma=\sigma(\gamma) b$, since $\psi(\sigma(\gamma))=\psi\left(\psi^{-1}(b \gamma)\right)=b \gamma$ and $\psi(\sigma(\gamma))=\sigma(\gamma) \psi(1)=\sigma(\gamma) b$.

We claim that $\sigma$ is a homomorphism of rings. We have $b\left(\gamma \gamma^{\prime}\right)=\sigma\left(\gamma \gamma^{\prime}\right) b$ and on the other hand $b\left(\gamma \gamma^{\prime}\right)=(b \gamma) \gamma^{\prime}=(\sigma(\gamma) b) \gamma^{\prime}=\sigma(\gamma)\left(b \gamma^{\prime}\right)=\sigma(\gamma)\left(\sigma\left(\gamma^{\prime}\right) b\right)=$ $\left(\sigma(\gamma) \sigma\left(\gamma^{\prime}\right)\right) b$. Hence, $\sigma\left(\gamma \gamma^{\prime}\right)=\sigma(\gamma) \sigma\left(\gamma^{\prime}\right)$.

It is clear that $\sigma\left(\gamma+\gamma^{\prime}\right)=\sigma(\gamma)+\sigma\left(\gamma^{\prime}\right)$. We have that $\sigma\left(e_{i}\right)=e_{i}$, since $e_{i} b=b e_{i}$ for all $i$. Hence $\sigma$ is a homomorphism of rings.

Define $\mu: \Lambda \rightarrow B$ by $\mu(\lambda)=b \lambda$, which is an isomorphism of right $\Lambda$-modules. Define $\tau: \Lambda \rightarrow \Lambda$ by letting $\tau(\gamma)=\mu^{-1}(\gamma b)$ for $\gamma$ in $\Lambda$. As above it can be shown that $\tau$ is an algebra homomorphism. Moreover, $b \gamma=\sigma(\gamma) b=b \tau(\sigma(\gamma))$, so that $\operatorname{id}_{\Lambda}=\tau \sigma$. Similarly we infer that $\operatorname{id}_{\Lambda}=\sigma \tau$. Hence $\sigma$ is an algebra automorphism.

Let $B^{\prime}={ }_{1} \Lambda_{\sigma}$ be the $\Lambda, \Lambda$-bimodule where the right multiplication is given by $\lambda * x=\lambda \sigma^{-1}(x)$. Define $\eta: B^{\prime} \rightarrow B$ by $\eta(1)=b$. This map is an isomorphism of $\Lambda, \Lambda$-bimodules, and hence $\Omega_{\Lambda^{e}}^{n}(\Lambda) \simeq{ }_{1} \Lambda_{\sigma}$. Thus (a) implies (b).

We now prove the converse, so assume (b) holds. For each right $\Lambda$-module $M$ we denote by $M_{\sigma}$ the right $\Lambda$-module $M \otimes_{\Lambda} \Omega_{\Lambda^{e}}^{n}(\Lambda)$. Let $S$ be a simple right $\Lambda$-module. From our initial remark concerning minimal resolutions, $\Omega_{\Lambda}^{n}(S) \simeq S \otimes_{\Lambda} \Lambda_{\sigma}$. Since $e_{i} \Lambda \simeq\left(e_{i} \Lambda\right)_{\sigma}$ and $e_{i} \mathfrak{r} \simeq\left(e_{i} \mathfrak{r}\right)_{\sigma}$ via the automorphism $\sigma$, it follows that $S \otimes_{\Lambda 1} \Lambda_{\sigma} \simeq S$. Hence $\Omega_{\Lambda}^{n}(S) \simeq S$ and all simple $\Lambda$-modules are $\Omega$-periodic.

Finally, we show that $\Lambda$ is selfinjective. Assume that $\Omega_{\Lambda^{e}}^{n}(\Lambda) \simeq{ }_{1} \Lambda_{\sigma}$. For any automorphism $\rho$ of $\Lambda$ and any left $\Lambda$-module $M$ we denote by ${ }_{\rho} M$ the left $\Lambda$-module ${ }_{1} \Lambda_{\rho} \otimes_{\Lambda} M$. As left $\Lambda$-modules $\Lambda$ and ${ }_{\sigma^{-1}} \Lambda$ are isomorphic via the automorphism $\sigma^{-1}$. Hence $D(\Lambda)$ and $D\left(\sigma_{\sigma^{-1}} \Lambda\right)$ are isomorphic as right $\Lambda$-modules. The map $\alpha: \operatorname{Hom}_{\Lambda}\left({ }_{1} \Lambda_{\sigma}, \Lambda\right) \rightarrow \Lambda$ given by $\alpha(f)=f(1)$ for $f$ in $\operatorname{Hom}_{\Lambda}\left({ }_{1} \Lambda_{\sigma}, \Lambda\right)$ gives an isomorphism between the left $\Lambda$-modules $\operatorname{Hom}_{\Lambda}\left({ }_{1} \Lambda_{\sigma}, \Lambda\right)$ and ${ }_{\sigma^{-1}} \Lambda$, where the left module structure of the former is given by the twisted bimodule structure of ${ }_{1} \Lambda_{\sigma}$. Since $D(\Lambda) \otimes_{\Lambda 1} \Lambda_{\sigma} \simeq D \operatorname{Hom}_{\Lambda}\left({ }_{1} \Lambda_{\sigma}, \Lambda\right) \simeq D\left({ }_{\sigma^{-1}} \Lambda\right)$, it follows that $D(\Lambda) \otimes_{\Lambda 1} \Lambda_{\sigma} \simeq D(\Lambda)$. Hence the injective cogenerator $D(\Lambda)$ of $\bmod \Lambda$ is a submodule of the projective right $\Lambda$-module $D(\Lambda) \otimes_{\Lambda} P^{n-1}$, so that $D(\Lambda)$ is also projective and $\Lambda$ is selfinjective. This completes the proof.

Remark. We remark that this theorem remains true when the hypotheses are weakened to $\Lambda$ being a flat artin algebra over a commutative local artinian ring $R$ such that $\Lambda / \mathfrak{r}$ is a separable $R$-algebra.

Note that in the proof of $\Lambda$ being selfinjective in Theorem 1.4 the fact that the automorphism $\sigma$ acts as the identity on a complete set of orthogonal idempotents was never used. Hence we have the following more general version, which we use in Theorem 1.6 .

Lemma 1.5. Let $K$ be an algebraically closed field, and let $\Lambda$ be a finite dimensional indecomposable $K$-algebra. Suppose that there exists a natural number $n$ and an automorphism $\sigma$ of $\Lambda$ such that $\Omega_{\Lambda^{e}}^{n}(\Lambda) \simeq{ }_{1} \Lambda_{\sigma}$. Then $\Lambda$ is selfinjective. 
Theorem 1.6. Let $K$ be an algebraically closed field. Let $\Lambda$ be a finite dimensional indecomposable $K$-algebra such that there is some natural number $n$ and some automorphism $\sigma$ of $\Lambda$ such that $\Omega_{\Lambda^{e}}^{n}(\Lambda) \simeq{ }_{1} \Lambda_{\sigma}$. Then

$$
\mathrm{HH}^{*}(\Lambda) / \mathcal{N} \simeq\left\{\begin{array}{l}
K, \text { or } \\
K[x]
\end{array}\right.
$$

where $\mathcal{N}$ is the ideal in $\mathrm{HH}^{*}(\Lambda)$ generated by the homogeneous nilpotent elements.

Proof. Since $K$ is algebraically closed we may assume that $\Lambda=K \mathcal{Q} / I$ where $\mathcal{Q}$ is the quiver of $\Lambda$ and $I$ is an admissible ideal.

If there is some natural number $m$ such that $\Omega_{\Lambda^{e}}^{m}(\Lambda)$ is isomorphic to $\Lambda$ as $\Lambda^{e}$ modules, then by Proposition 1.1 we have $\mathrm{HH}^{*}(\Lambda) / \mathcal{N} \simeq K[x]$, with $x$ in degree $m$ when $m$ is chosen minimal. In particular this occurs when $\sigma$ has finite order.

Suppose now that $\Omega_{\Lambda^{e}}^{m}(\Lambda) \not \Lambda$ as $\Lambda^{e}$-modules for any $m$, and in particular $\sigma$ has infinite order.

Let $\eta: \Omega_{\Lambda^{e}}^{n p}(\Lambda) \rightarrow \Lambda$ represent an element in $\operatorname{HH}^{n p}(\Lambda)$ (with $p \geq 1$ ). By assumption $\eta$ is not an isomorphism. From Lemma 1.5, $\Lambda$ is selfinjective, and thus, for any $i \geq 1, \Omega_{\Lambda^{e}}^{i n p}(\Lambda) \simeq{ }_{1} \Lambda_{\sigma^{i p}}$, which is an indecomposable $\Lambda^{e}$-module, and $\Omega_{\Lambda^{e}}^{(i-1) n p}(\eta): \Omega_{\Lambda^{e}}^{i n p}(\Lambda) \rightarrow \Omega_{\Lambda^{e}}^{(i-1) n p}(\Lambda)$ is not an isomorphism. Since all the modules $\left\{\Omega_{\Lambda^{e}}^{i n p}(\Lambda)\right\}_{i \geq 0}$ have bounded length, there exists, by Harada-Sai (see [2]), some natural number $N$ such that the composition $\Omega_{\Lambda^{e}}^{N n p}(\eta) \cdots \Omega_{\Lambda^{e}}^{2 n p}(\eta) \Omega_{\Lambda^{e}}^{n p}(\eta) \eta$ is zero. Hence $\eta$ is nilpotent.

Now let $\eta: \Omega_{\Lambda^{e}}^{s}(\Lambda) \rightarrow \Lambda$ represent an element in $\operatorname{HH}^{s}(\Lambda)$ where $s \geq 1$ and $n \backslash>s$. Let $r$ be such that $r s=q n$ for some $q$. Then $\eta^{r} \in \mathrm{HH}^{q n}(\Lambda)$. By the above argument, $\eta^{r}$ is nilpotent and hence $\eta$ is nilpotent.

Thus any homogeneous element in $\mathrm{HH}^{\geq 1}(\Lambda)$ is nilpotent. Hence $\mathrm{HH}^{*}(\Lambda) / \mathcal{N} \simeq$ $K$. This completes the proof of the theorem.

Corollary 1.7. Let $K$ be an algebraically closed field, and let $\Lambda$ be a finite dimensional selfinjective $K$-algebra of finite representation type. Suppose that $\Lambda$ is indecomposable and not semisimple. Then all indecomposable $\Lambda$-modules are $\Omega$ periodic or projective, and there exists a natural number $n$ and an automorphism $\sigma$ of $\Lambda$ such that $\Omega_{\Lambda^{e}}^{n}(\Lambda) \simeq{ }_{1} \Lambda_{\sigma}$. Moreover

$$
\mathrm{HH}^{*}(\Lambda) / \mathcal{N} \simeq\left\{\begin{array}{l}
K, \text { or } \\
K[x]
\end{array}\right.
$$

where $\mathcal{N}$ is the ideal in $\mathrm{HH}^{*}(\Lambda)$ generated by the homogeneous nilpotent elements.

Proof. Since $\Lambda$ is selfinjective, $\Omega_{\Lambda}^{i}(M)$ is indecomposable for all $i$ and all indecomposable non-projective $\Lambda$-modules $M$. As $\Lambda$ is of finite representation type, there must exist positive integers $i_{M}$ and $1 \leq r_{M}$ for any indecomposable non-projective $\Lambda$-module $M$ such that $\Omega_{\Lambda}^{i_{M}}(M) \simeq \Omega_{\Lambda}^{i_{M}+r_{M}}(M)$. Hence $\Omega_{\Lambda}^{r_{M}}(M) \simeq M$ for all indecomposable non-projective $\Lambda$-modules $M$ and some integer $r_{M}$. Moreover, since $\Lambda$ is indecomposable, all simple modules are non-projective. The second part of the claim follows directly from Theorems 1.4 and 1.6.

We remark that it is not known whether the automorphism $\sigma$ of Theorem 1.4 is necessarily of finite order, though it is so for $\Lambda$ of finite representation type $A_{n}$. 


\section{REFERENCES}

1. Asashiba, H., The derived equivalence classification of representation-finite self-injective algebras, J. Algebra 214 (1999), 182-221. MR 2000g:16019

2. Auslander, M., Reiten, I. and Smalø, S. O., Representation theory of artin algebras, Cambridge Studies in Advanced Mathematics 36, Cambridge University Press, 1995. MR 96c:16015

3. Benson, D., Representation theory and cohomology II: Cohomology of groups and modules, Cambridge Studies in Advanced Mathematics 31, CUP, 1991. MR 93g:20099

4. Brenner, S. and Butler, M.C.R., Almost periodic algebras and pivoted bimodules: resolutions and Yoneda algebras, preprint 2000.

5. Erdmann, K. and Holm, T., Twisted bimodules and Hochschild cohomology for self-injective algebras of type $A_{n}$, Forum Math. 11 (1999), 177-201. MR 2001c:16018

6. Erdmann, K., Holm, T. and Snashall, N., Twisted bimodules and Hochschild cohomology for self-injective algebras of type $A_{n}$ II, Algebras and Representation Theory 5 (2002), 457-482.

7. Erdmann, K. and Snashall, N., On the Hochschild cohomology of preprojective algebras I, II, J. Algebra 205 (1998), 391-412, 413-434. MR 99e:16013

8. Erdmann, K. and Snashall, N., Preprojective algebras of Dynkin type: periodicity and the second Hochschild cohomology, Canad. Math. Soc. Conference Proceedings 24 (1998), 183193. MR 99h:16016

9. Happel, D., Hochschild cohomology of finite-dimensional algebras, Springer Lecture Notes in Mathematics 1404 (1989), 108-126. MR 91b:16012

10. Membrillo-Hernández, F.H., Homological properties of finite-dimensional algebras, D.Phil. Thesis, University of Oxford (1993).

11. Riedtmann, C., Representation-finite self-injective algebras of class $A_{n}$, In: Representation Theory II, Proc. Second Internat. Conf., Carleton Univ., Ottawa, Springer Lecture Notes in Mathematics 832 (1979), 449-520. MR 82k:16040

12. Scherzler, E. and Waschbüsch, J., A class of self-injective algebras of finite representation type, In: Representation Theory II, Proc. Second Internat. Conf., Carleton Univ., Ottawa, Springer Lecture Notes in Mathematics 832 (1979), 545-572. MR 82i:16034

Department of Mathematics, Virginia Tech, Blacksburg, Virginia 24061-0123

E-mail address: green@math.vt.edu

Department of Mathematics and Computer Science, University of Leicester, UniverSity RoAd, LEICESTER, LE1 7RH, ENGLAND

E-mail address: N.Snashall@mcs.le.ac.uk

Institutt for matematiske fag, NTNU, N-7491 Trondheim, Norway

E-mail address: oyvinso@math.ntnu.no 